\title{
Classicality without Decoherence: A Reply to Schlosshauer
}

\author{
Leslie Ballentine
}

Received: 8 August 2008 / Accepted: 22 September 2008 / Published online: 3 October 2008

(C) The Author(s) 2008. This article is published with open access at Springerlink.com

\begin{abstract}
Schlosshauer has criticized the conclusion of Wiebe and Ballentine (Phys. Rev. A 72:022109, 2005) that decoherence is not essential for the emergence of classicality from quantum mechanics. I reply to the issues raised in his critique, which range from the interpretation of quantum mechanics to the criterion for classicality, and conclude that the role of decoherence in these issues is much more restricted than is often claimed.
\end{abstract}

Keywords Classicality in quantum mechanics - Decoherence $\cdot$ Interpretation of state vector

\section{Introduction}

In his paper [1], Schlosshauer raises some important questions about the emergence of classical behavior from quantum mechanics (QM) and the appropriate criterion for effective classicality. These are related to the broader issues of the meaning and interpretation of the state vector in QM, and to the narrower issue of the role of decoherence in these problems. There is a variety of opinions regarding these fundamental questions, and also some wide-spread misconceptions about them. Therefore I shall discuss these issues broadly, rather than presenting a paragraph-by-paragraph reply to Schlosshauer's paper.

There is no doubt that decoherence plays a very important practical role in any experiment that depends critically on the maintenance of quantum coherence. Indeed, a large fraction of the effort in designing qubits for quantum computation is devoted to the control of decoherence. But the situation is quite different when considering conceptual problems in the foundations of QM, such as Schrödinger's cat paradox, the problem of measurement, and the emergence of classicality from QM. There, the

L. Ballentine $(\bowtie)$

Simon Fraser University, Burnaby, BC V5A1S6, Canada

e-mail: ballenti@sfu.ca 
usefulness of decoherence is much more limited, and in some cases, is nonexistent. I explain these matters in this paper.

\section{Schrödinger's Cat and the Measurement Problem}

The key problem is illustrated by the so-called cat paradox, introduced by Schrödinger in 1935. A cat is placed in a device with an unstable atom. When the atom decays, a poison is released which kill the cat. The state vector of the atom-plus-cat system will have the form

$$
\alpha_{1}\left|a_{1}\right\rangle\left|c_{1}\right\rangle+e^{i \phi} \alpha_{2}\left|a_{2}\right\rangle\left|c_{2}\right\rangle
$$

Here the vectors $\left|a_{1}\right\rangle$ and $\left|a_{2}\right\rangle$ denote an undecayed and a decayed atom, respectively, and $\left|c_{1}\right\rangle$ and $\left|c_{2}\right\rangle$ denote a live and a dead cat. The live and dead eigenvectors are, of course, very highly degenerate, although the simplified notation does not so indicate. Hence the two terms in (1) are to be interpreted as the projections of the state vector onto the live and the dead subspaces of the infinite dimensional Hilbert space. The amplitude $\alpha_{1}$ decreases with time (approximately exponentially), while $\alpha_{2}$ increases with time. However, their precise time dependence is not important, since we shall only be discussing the meaning of this state vector at a fixed time. Note the inclusion of a relative phase $e^{i \phi}$, which is often omitted, but which will be useful in the discussion.

Evidently, this state is a coherent superposition of macroscopically distinct components. Whether this fact is innocuous or alarming depends on the interpretation that one adopts for the state vector, as will be discussed in the next section.

The above state vector does not take account of the environment. If there were no interaction between the atom-plus-cat system and the environment, then we should simple multiply (1) by an environmental state $|e\rangle$, and this factor would be irrelevant to both the predictions from and the interpretation of the state vector. But when the (weak, but unavoidable) interaction between the system and the environment is taken into account, we obtain a state vector of the form

$$
\alpha_{1}\left|a_{1}\right\rangle\left|c_{1}\right\rangle\left|e_{1}\right\rangle+e^{i \phi} \alpha_{2}\left|a_{2}\right\rangle\left|c_{2}\right\rangle\left|e_{2}\right\rangle
$$

where $\left|e_{1}\right\rangle$ and $\left|e_{2}\right\rangle$ are states of the environment. This is also a coherent superposition of macroscopically distinct components. Indeed, if the presence of a macroscopic superposition is regarded as alarming, then the inclusion of the environmental interaction only makes the problem more severe, since we now have an even more macroscopic superposition involving states of the environment. Thus environmental effects (known as decoherence) are of no value at all in resolving Schrödinger's cat paradox.

The so-called problem of measurement is isomorphic to the cat paradox. In the above equations, we merely interpret the vectors $\left|a_{i}\right\rangle$ as describing an observable of the microscopic object that we wish to measure, and $\left|c_{j}\right\rangle$ as a pointer position eigenstate of the apparatus. (There may also be more than two terms in the sums in (1) and (2).) The state vector (2) is seen to be a coherent superposition of macroscopically distinct pointer positions. But at the end of a measurement, we observe a single value 
of the pointer-position, so how does the observed value arise from the superposition state that QM gives us? Once again, this problem is easy or impossible to solve, depending upon the interpretation of the state vector that is adopted. As with the cat paradox, decoherence proves to be of no value in resolving the problem. I have discussed the measurement problem in more detail in [2] and [3].

Although the prediction by QM of a coherent superposition of macroscopically distinct components is unambiguous and unavoidable, with or without decoherence, there remains the question of observability of this coherence. I deal with that aspect in Sect. 4.

\section{Ensemble vs Individual Interpretations of the State Vector}

The cat paradox and the measurement problem may appear innocuous or difficult, depending on the interpretation that one gives to the state vector. Indeed, Schlosshauer suggests that different assumptions-an ensemble vs an individual interpretationmay be at the root of the disagreement between Wiebe and myself [4] and Zurek [5] regarding the origin of classical behavior in macroscopic objects. Let us consider these two interpretations,

Ensemble interpretation: A state vector describes the statistical properties of an ensemble of similarly prepared systems.

Individual interpretation: A state vector $|\Psi\rangle$ provides a complete description of an individual system. In particular, a dynamical variable $Q$ has a value $(q$, say) if and only if $Q|\Psi\rangle=q|\Psi\rangle$.

Other, more nuanced interpretations are possible, but for simplicity I consider only these two.

By way of motivation, it is well known that QM is an irreducibly statistical theory. QM does not describe the phenomena, but only the probability distribution of the possible phenomena. QM does not predict the result of a measurement, but only the probabilities of the possible results of that measurement. It is, therefore, natural to say that the QM state vector describes an ensemble of similarly prepared systems. The collected results of measurements on this ensemble allow one to compare the probabilities that are predicted by QM with observation.

A few clarifying remarks are in order. First, the ensemble does not consist of the component terms in (1) or (2). It is the ensemble of similarly prepared systems. Second, the state vector is not regarded as an element of reality. Its significance is in yielding the probability distributions of all observables, not merely those probabilities that are obtained from the absolute squares of the amplitudes in the separate terms of (1) or (2).

I can say very little in defense of the individual interpretation, except that it used to be widely held, and still has a significant number of adherents. According to the individual interpretation, the cat in (1) or (2) is neither alive nor dead, and the pointer of the measurement apparatus has no definite value, until some external classical (i.e. not quantum mechanical) agent intervenes to "collapse" the state vector. But there is no such agent! (In anticipation of arguments yet to come, any suggestion that decoherence might achieve the same result that would be obtained from the ad hoc 
"collapse" postulate is unjustified.) The hypothetical "collapse" is incompatible with the mathematical formalism of QM, and can even lead to incorrect results [6]. QM is (assumed to be, until evidence to the contrary is found) the fundamental theory, to which classical mechanics is only an approximation of limited accuracy. The individual interpretation, at least in the form given above, is untenable. Decoherence is irrelevant to this conclusion.

Neither the cat paradox nor the measurement problem are serious for the ensemble interpretation, since it predicts all of the correct probabilities.

However, I am unsure as to whether the choice between an ensemble or an individual interpretation is at the root of our disagreement with Zurek, as was suggested by Schlosshauer. I have not noticed the question of an ensemble vs an individual interpretation being a prominent feature in his papers on decoherence theory.

\section{Observability of Coherence}

Coherence is observed through interference. Any effect that depends on the phase factor $e^{i \phi}$ in (1) or (2) is an interference effect. Clearly the live or dead condition of the cat, or the probability distribution of the pointer position in the measurement problem, does not depend on the phase, and so cannot distinguish between a coherent superposition and an incoherent mixture. So how could we detect the coherence?

Before answering that question, I must point out that the phrase "coherent superposition of pointer positions" contains an ambiguity. It suggests a state vector of the form

$$
\alpha_{1}\left|c_{1}\right\rangle+e^{i \phi} \alpha_{2}\left|c_{2}\right\rangle
$$

This form may be called first order coherence. It does not exist in the problems that we have considered so far, but if it exists in other cases, it could be revealed by a measurement involving only the system described by $\left|c_{j}\right\rangle$. In contrast, (1) exhibits second order coherence, which can be revealed only through the correlations between the systems described by $\left|a_{i}\right\rangle$ and $\left|c_{j}\right\rangle$. In turn, (2) exhibits third order coherence, which manifests itself as a correlation between the compound system (whose basis vectors are the products $\left|a_{i}\right\rangle\left|c_{j}\right\rangle$ ) and the environment. The term decoherence is, therefore, misleading, because the effect of the environment is not to destroy coherence, but rather to transform it into a higher order form of entanglement.

It has sometimes been suggested that to detect the first order coherence in the state (3), which describes a superposition of positions, one would have to perform a projective measurement onto nonlocal states such as $\left|c_{1}\right\rangle+\left|c_{2}\right\rangle$. But that would require preparing the appropriate apparatus in such a nonlocal state, and so would beg the question of whether such nonlocal states of macroscopic objects really exist. On the contrary, the way to detect the coherence in (3) is to measure some observable that does not commute with the position, such as the momentum. Its probability distribution contains an interference pattern that does depend on the phase $\phi$. Similarly, the second and third order coherences in (1) and (2) can, in principal, be detected by measuring the correlations among observables that are not diagonal in the basis formed by the set of vectors $\left\{\left|a_{i}\right\rangle\left|c_{j}\right\rangle\left|e_{k}\right\rangle\right\}$. Nor is it necessarily the case that higher order coherences are more difficult to detect that those of lower order. There are many examples 
in which second order coherence occurs in systems that lack first order coherence $[7,8]$.

However, it is unlikely that any of these coherences will be observable in macroscopic systems. The wavelength of the interference patterns would be smaller than the size of an elementary particle [7], and hence impossible to resolve. The effect of environmental decoherence would be to randomize the phase $\phi$ within the ensemble of repeated state preparations that is necessary to obtain statistical data. Thus the role of decoherence is to destroy an interference pattern that is unobservable anyhow.

\section{Classicality of Hyperion}

The fundamental quantities of classical mechanics are variables like positions and the orbits that they trace out in time. The fundamental quantities of QM are state vectors and self-adjoint operators that represent observables. It is not obvious just what structures in QM should lead to corresponding classical structures in the appropriate limit [9]. Since QM, at least in its present form, is an irreducibly statistical theory, it is natural to require that the quantum and classical probabilities should agree for all observables that have a classical analogue.

Schlosshauer objects that in [4] we showed a classical limit for only one component of the angular momentum. However, that component $\left(J_{z}\right)$ was chosen only as typical. We could have easily shown similar results for any component in any direction. One should also study more complicated observables such as $\left(J_{x} J_{y}+J_{y} J_{x}\right) / 2$. Although we have not done that for Hyperion, a study of such correlation observables was done for two similar systems: a dynamically driven spin [10], and two coupled rotors [11]. These were found to have a proper classical limit; moreover, in [10] it was also found that the QM average of a commutator, such as $\left\langle\left(J_{x} J_{y}-J_{y} J_{x}\right) / 2 i\right\rangle$, tended to zero in the classical limit. All of this was shown without needing to include decoherence. There is, therefore, a growing body of examples that show how a proper classical limit may be obtained for isolated systems, without the need to invoke decoherence. The inclusion of decoherence makes only small quantitative corrections, and is not needed as a matter of principle.

Schlosshauer points out that in an isolated system there is always one observable that will not have a classical limit, namely the projection operator $|\Psi\rangle\langle\Psi|$, where $|\Psi\rangle$ is the state vector. That is correct. If QM is the true theory, then there are no exactly classical systems. So when we say that a macroscopic system behaves classically, we should follow the terminology of John Bell, and say that it behaves classically FAPP (for all practical purposes)! But the state vector (2), which includes the environment, also has a similar nonclassical projection operator, so once again decoherence makes no difference to questions of principle.

Finally, I come to what may indeed be the root cause of our disagreement. If one takes a pure state, like (2), which includes the environment, forms the corresponding density matrix, and traces over the environment, then the resulting (reduced) density matrix for the remaining system will be a mixed state. Schlosshauer claims that this mixture will be equivalent to an "ensemble of narrow position-space wave packets." There are many problems with this assertion. First, it is merely asserted. No proof, 
or reference to a proof, is given, and I have never seen one that I consider to be convincing. Second, it is well known that any mixed state can be formally represented as a mixture of pure states in infinitely many ways. No one of these formal mixture representations has any privileged physical significance. The possible mixture representations of a given non-pure density matrix have been classified $[12,13]$. In order to prove what Schlosshauer has asserted, it would be necessary to apply the classification theory and to show (if true) that all possible mixture representations are in terms of narrow wave packets. I doubt that this will be true. Lastly, even if Schlosshauer's assertion turns out to be correct, it will only confirm that the ensemble interpretation of QM holds into the classical limit, i.e. that the classical limit of a quantum state is an ensemble of classical orbits (with a predicted probability distribution), and not a single classical orbit. But that is just what I have argued in this and several other papers, which reach that conclusion without needing to invoke decoherence.

\section{Conclusions}

In this paper I have tried to clear up some common misconceptions about the scope and limitations of the decoherence program. Decoherence and its avoidance are very important in interference experiments, and so decoherence theory has proven to be very useful in practical matters. But the claims for decoherence theory in the conceptual problems of QM have been exaggerated. Decoherence theory is of no help at all in resolving Schrödinger's cat paradox or the problem of measurement. Its role in establishing the classicality of macroscopic systems is much more limited than is often claimed. It succeeds in eliminating certain interference patterns in macroscopic systems, but these are anyhow much too fine to be observed. The claim that the environment will decohere an extended wave function into a mixture of localized wave packets remains unproven. Even if that claim were (improbably) proven correct, it would only confirm the ensemble interpretation of quantum states, and would not restore a version of the individual interpretation.

As was said in [4], "It is not correct to assert that environmental decoherence is the root cause of the appearance of the classical world."

Open Access This article is distributed under the terms of the Creative Commons Attribution Noncommercial License which permits any noncommercial use, distribution, and reproduction in any medium, provided the original author(s) and source are credited.

\section{References}

1. Schlosshauer, M.: Found. Phys. 38, 796 (2008)

2. Ballentine, L.E.: Rev. Mod. Phys. 42, 358-381 (1970)

3. Ballentine, L.E.: Quantum Mechanics: A Modern Development. World Scientific, Singapore (1998)

4. Wiebe, N., Ballentine, L.E.: Phys. Rev. A 72, 022109 (2005)

5. Zurek, W.H.: Phys. Scripta T 76, 186 (1998)

6. Ballentine, L.E.: Found. Phys. 20, 1329-1343 (1990)

7. Silverman, M.P.: Quantum Superpositions. Springer, Berlin (2008)

8. Ballentine, L.E.: In: Quantum Mechanics: A Modern Development, pp. 221-223. World Scientific, Singapore (1998) 
9. Ballentine, L.E.: In: Quantum Mechanics: A Modern Development. World Scientific, Singapore (1998). Chapter 14

10. Ballentine, L.E.: Phys. Rev. A 47, 2592-2600 (1993)

11. Ballentine, L.E.: Phys. Rev. A 70, 032111 (2004)

12. Hughston, L.P., Joza, R., Wooters, W.K.: Phys. Let. A 183, 14-18 (1993)

13. Kirkpatrick, K.A.: Found. Phys. 19, 95-102 (2006) 